\title{
Population Synthesis of Type Ia SNe: Constraining Free Parameters from Observations
}

\author{
Maxwell Moe \& Rosanne Di Stefano \\ Harvard-Smithsonian Center for Astrophysics \\ 60 Garden Street, Cambridge, MA, 02138, USA \\ email: mmoe@cfa.harvard.edu
}

\begin{abstract}
Computing the rate of Type Ia supernovae (SNe Ia) from first principles is difficult because there are large uncertainties regarding several key binary processes such as common envelope evolution, tidal interactions, and the efficiency of mass transfer. Fortunately, a range of observational parameters of binaries in intermediate stages of evolution can help us model these processes in a way that is likely to mirror the true binary evolution. We discuss how this observationally-motivated approach may have the effect of increasing the predicted rate of single degenerate progenitors of SNe Ia, while simultaneously decreasing the number of double degenerate progenitors.
\end{abstract}

Keywords. stars: AGB and post-AGB, white dwarfs, supernovae - binaries: close, symbiotic

\section{Introduction}

The predicted rates and delay time distribution of SNe Ia from single degenerate (SD) and double degenerate (DD) scenarios are typically compared to those inferred from observations to determine which channel is dominant (Maoz et al. 2010). Recent population synthesis studies appear to favor the DD model because the modeled SD rate underpredicts the observed rate, especially at late delay times (Ruiter et al. 2009). However, there are dozens of different prescriptions and free parameters invoked in binary population synthesis (see Nelemans et al., these proceedings), and the predicted SN Ia rate is highly dependent on the adopted models and formalisms.

The three-dimensional phase space of orbital periods, donor masses, and white dwarf (WD) masses has been studied in detail to determine which systems subsequently evolve toward SNe Ia (e.g. Kato, these proceedings). Although these progenitor "islands" vary in the literature, the most popular evolutionary pathways include stable Roche-lobe overflow (RLOF) from a main-sequence (MS) or slightly evolved subgiant donor (WD+MS channel), stable RLOF from a red giant branch (RGB) donor (WD+RGB channel), wind accretion from an RGB or early asymptotic giant branch (AGB) donor (symbiotic channel), and the merging of two carbon-oxygen (CO) WDs exceeding the Chandrasekhar mass (canonical DD channel). We crudely project these evolutionary channels onto the one dimensional vector of orbital periods in Figure 1. In short, SD channels occupy a wide parameter space while $\mathrm{DD}$ progenitors must emerge at $\mathrm{P} \sim 200$ days after the first phase of binary evolution so that a common envelope (CE) occurs on the AGB during the second phase, leading to a close pair of CO WDs.

Equally important to delineating these progenitor islands is understanding how the initial MS+MS binaries map into and populate this phase space of systems. In this proceeding, we discuss a few aspects of this initial stage of binary evolution in which the physical processes remain poorly understood, but where various observations can significantly constrain the parametrization. 


\section{First Phase of Binary Evolution}

Stable RLOF. The fraction of mass lost by a subgiant donor that is retained by a MS accretor via RLOF, $\beta$, is not well quantified, but the adopted prescription has significant consequences on the predicted rates of SD and DD SNe Ia. For example, Mennekens et al. (2010) showed that for conservative mass transfer, i.e. $\beta=1$, the RLOF pathway can dominate the DD rate compared to the standard channel whereby the DD progenitor passes through two phases of CE. However, they also showed that slightly decreasing $\beta$ dramatically reduces the DD rate.

In order to constrain $\beta$, we appeal to the recent observations and modeling of Algol binaries by van Rensbergen et al. (2011). They found that conservative mass transfer could not reproduce the observed period or mass-ratio distribution of Algols, while their liberal evolution models more closely matched the observations. Using the non-conservative RLOF formalism of Hurley et al. (2002) that incorporates the thermal timescales of the donor and accretor, we show in Figure 1 how changing $\beta$ from 1 (dashed green) to the observationally motivated prescription (solid green) affects the final period distribution. Fewer systems can sufficiently widen to produce CO WDs at long periods $\mathrm{P} \sim 200$ days, while the majority of binaries either merge, produce He WDs, or leave C/O WDs at shorter periods. Therefore, applying the observationally motivated non-conservative mass transfer formalism dramatically reduces the DD rate but only slightly enhances the $\mathrm{WD}+\mathrm{RGB}$ and symbiotic SD rates.

Common Envelope Evolution. To calculate the final post-CE orbital separation, we adopt the energy formalism of Webbink (1984), where the product of the efficiency $\alpha_{\mathrm{CE}}$ and binding energy $\lambda$ parameters is to be constrained by observations of post-CE binaries. The value of $\alpha_{C E} \lambda$ has significant consequences on the rate of SNe Ia. For example, Ruiter et al. (2009) found that decreasing $\alpha_{C E} \lambda$ from 1.0 to 0.5 decreased the expected $\mathrm{DD}$ rate by a factor of $\sim 2$ while increasing the predicted SD rate by a factor of $\sim 3$. However, the period distributions of post-CE WD+MS binaries (Rebassa-Mansergas et al. 2008) and close binary central stars of planetary nebulae (Miszalski et al. 2009) peak at $\mathrm{P} \sim 1$ day, indicating an even lower efficiency $\alpha_{C E} \lambda \sim 0.1$ for typical mass-ratios. Moreover, reconstruction of the initial binary configurations of observed post-CE systems also suggest lower efficiencies $\alpha_{C E} \lambda \sim 0.15$ for typical mass ratios $\mathrm{q}=\mathrm{M}_{2} / \mathrm{M}_{\text {giant }} \gtrsim 0.3$, but the presence of post-CE systems with brown dwarf secondaries requires $\alpha_{C E} \lambda$ to be much higher for lower mass ratios (De Marco et al. 2011).

In Figure 1, we show how changing the efficiency of CE from the canonical constant value (dashed blue) to a variable that increases with decreasing mass ratio (solid blue) as constrained by these recent observations affects the period distribution of post-CE binaries. We note that in addition to translating systems toward smaller separations, the anti-correlation between $\alpha_{C E}$ and q creates a narrow peak at short periods, consistent with observations. The increased number of predicted post-CE binaries at short periods dramatically enhances the WD+MS and WD+RGB SD rates while the decreased number at intermediate periods dramatically reduces the DD rate.

Tidally Enhanced Mass Loss. Tides can enhance the mass loss rate of the donor and direct much of the mass-losing wind toward the accreting companion. There exists several lines of observational evidence for equatorially enhanced mass loss due to tides. First, binaries that have inverted their mass ratios long before the donor fills its Roche lobe are testament to efficient tidal mass loss enhancement (Tout \& Eggleton 1988). Second, the observed eccentricity-period distributions of barium stars and post-AGB binaries require the donor AGB star to expel a large fraction of its envelope onto the companion before the orbit can circularize (Karakas et al. 2000). Finally, the observed wind 
structure of Mira necessitates a new mode of wind accretion, i.e. wind-RLOF, to exceed the spherically symmetric Bondi-Hoyle approximation by an order of magnitude (Mohamed \& Podsiadlowski 2011). To model this interplay between tides, enhanced mass loss, and wind accretion, we rely on these observations, standard tidal theory to determine the spin-up rate of a giant due to tidal torques from its companion, and the rotating AGB models of Dorfi \& Hoefner (1996) to calculate the degree of mass loss enhancement and the fraction that is directed into the equatorial plane.

In Figure 1, we compare our predicted period distributions for systems that avoided RLOF/CE and experienced only wind accretion assuming no tidal mass-loss enhancement (dashed red) and with tidal mass-loss enhancement (solid red) as motivated by the observations. Because tidally enhanced winds can expel the envelope of a giant before the giant can expand to its full dimensions had it not lost significant mass, wind accreting non-circularized systems emerge at short periods $\mathrm{P} \sim 300-2,000$ days, consistent with the eccentricity-period distribution of barium stars (Karakas et al. 2000). Because more systems emerge at $\sim 1,000$ days, the symbiotic SNe Ia channel is relatively enhanced.

\section{Subsequent Evolution}

We can use these observational constraints of physical processes to model the second stage of binary evolution. For example, the discovery of extreme mass-ratio double WDs (Kilic et al. 2011) with progenitors $\mathrm{q} \sim 0.2$ also indicates a variable $\alpha_{C E}$ that is anticorrelated with the mass-ratio q. If we apply the same CE formalism to AGB + WD binaries as is constrained from post-CE binaries that emerged from the first phase of evolution, then the predicted post-CE period distribution of $\mathrm{CO} \mathrm{WD}+\mathrm{CO} \mathrm{WD}$ pairs will also be narrowly peaked at $\sim 0.8$ days. Only the few WD pairs in the short period tail of this distribution will merge within a Hubble time. Moreover, the shape of the period distribution is such that the predicted delay time distribution of $\mathrm{CO} \mathrm{WD}+\mathrm{CO} \mathrm{WD}$ pairs with total mass $M>M_{C h}$ will be flatter than originally predicted, i.e. weighted more toward late delay times.

Equatorially enhanced mass loss via tidal interactions in a symbiotic system will also increase the likelihood that the WD will accumulate sufficient mass to reach $\mathrm{M}_{\mathrm{Ch}}$. As discussed in Chen et al. (2011), tides will affect the mass loss evolution, allowing many giants to lose most of their envelope at $\sim 10^{-6} \mathrm{M}_{\odot} \mathrm{yr}^{-1}$; therefore, the mass incident on the WD will be accumulated instead of being ejected via nova eruptions or lost due to optically thick winds. Moreover, tides will direct the winds from the giant into the orbital plane, increasing the fraction of material that is incident onto the accreting WD (see Mohamed, these proceedings).

\section{Conclusions}

We have used observations of binaries in intermediate stages of evolution to motivate and constrain our prescriptions of various physical processes. We have demonstrated that the observationally constrained values of $\alpha_{C E} \lambda$ and $\beta$ leave binaries in closer orbits after the first phase of evolution, so that they are more likely to subsequently evolve toward $\mathrm{SD}$ SNe Ia (WD+MS or WD+RGB) rather than DD SNe Ia. The empirically derived anti-correlation between $\alpha_{C E}$ and q will also cause the CO WD + CO WD post-CE period distribution to peak at $\sim 0.8$ days, which further reduces the DD rate and shifts these mergers to later delay times. The inclusion of equatorially enhanced mass loss via tides, as evidenced by various kinds of binaries, allows more systems to avoid $\mathrm{CE}$ and emerge at periods $\mathrm{P} \sim 1,000$ days, so that they will more likely evolve toward symbiotic SD SNe Ia. Furthermore, incorporating tidal mass loss enhancement will allow accreting WDs in symbiotic systems to more readily approach the Chandrasekhar mass. 


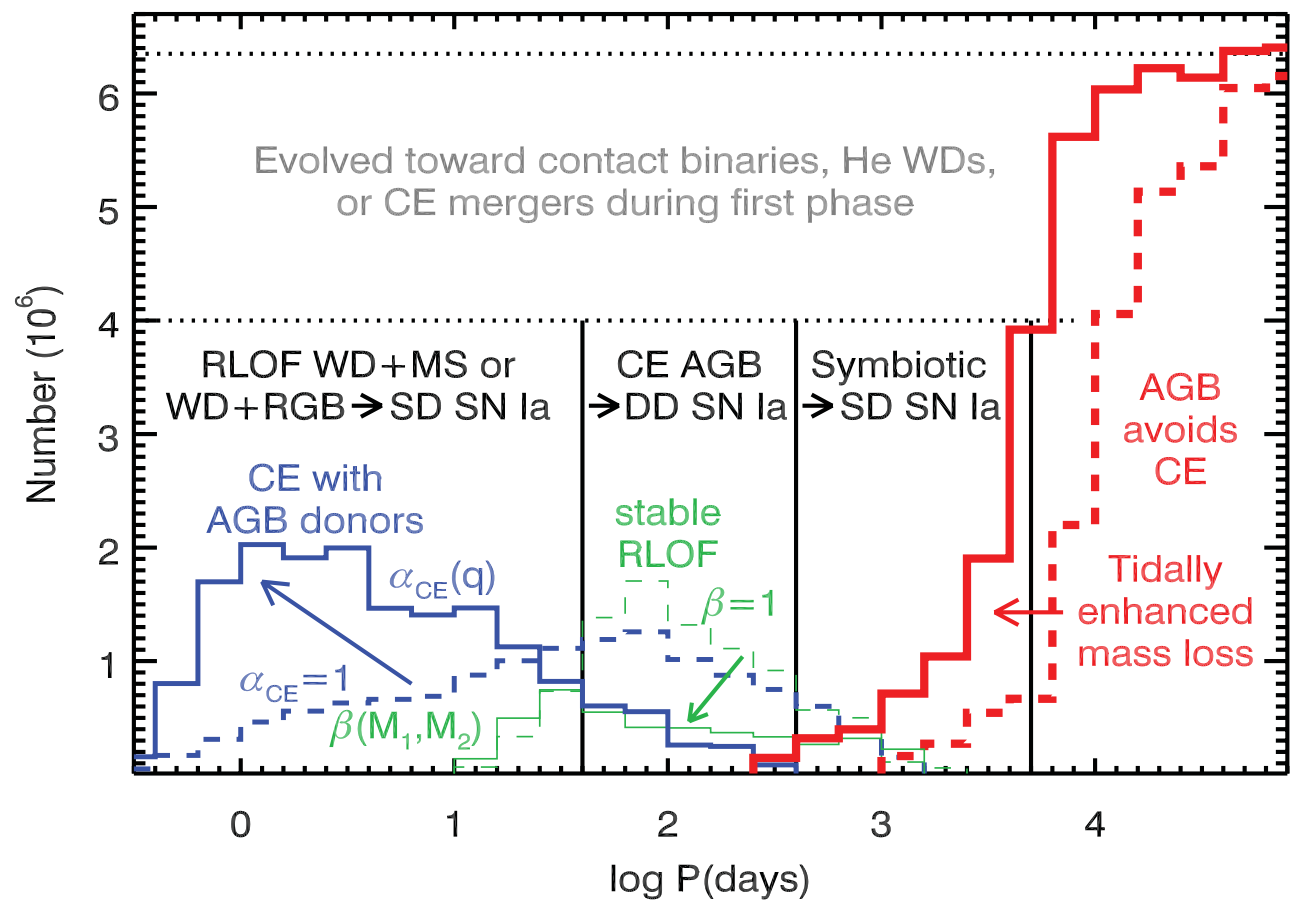

Figure 1. The predicted C/O WD + MS period distribution with $\mathrm{M}_{\text {total }}>2.0 \mathrm{M}_{\odot}$. We display how changing the descriptions of $\beta$ (thin green), $\alpha_{C E}$ (medium blue), and tidal mass loss enhancement (thick red) from the canonical values (dashed) to the values constrained by observations (solid) will affect the predicted period distribution. We indicate the subsequent evolution (separated by vertical lines), and in all three cases the observationally motivated prescriptions favor an increase in the progenitors of SD SNe Ia, typically at the expense of the DD channel.

MM acknowledges support by the National Science Foundation (NSF) Graduate Research Fellowship Program under Grant \#'s DGE-0644491 and DGE-0946799.

\section{References}

Chen, X., Han, Z., \& Tout, C. A., 2011, ApJ, 735, 31

De Marco, O., Passy, J.-C., Moe, M., Herwig, F. et al., 2011, MNRAS, 411, 2277

Dorfi, E. A. \& Hoefner, S., 1996, MNRAS, 313, 605

Karakas, A. I., Tout, C. A., \& Lattanzio, J. C., 2000, MNRAS, 316, 689

Kilic, M., Brown, W. R., Allende Prieto, C., Agüeros, M. A., et al., 2011, ApJ, 727, 3

Hurley, J. R., Tout, C. A., \& Pols, O. R., 2002, MNRAS, 329, 897

Maoz, D., Sharon, K., \& Gal-Yam, A., 2010, ApJ, 722, 1879

Mennekens, N., Vanbeveren, D., De Greve, J. P., \& De Donder, E., 2010, A\&A, 515, 89

Miszalski, B., Acker, A., Moffat, A. F. J., Parker, Q. A., \& Udalski, A., 2009, A\&A, 496, 813

Mohamed, S. \& Podsiadlowski, P., 2011, ASPC, 445, 355

Rebassa-Mansergas, A., Gänsicke, B. T., Schreiber, M. R., et al., 2008, MNRAS, 390, 1635

Ruiter, A. J., Belczynski, K., \& Fryer, C., 2009, ApJ, 699, 2026

Tout, C. A. \& Eggleton, P. P., 1988, MNRAS, 231, 823

van Rensbergen, W., de Greve, J. P., Mennekens, N., et al., 2011, A $\& A, 528,16$

Webbink, R. F., 1984, ApJ, 227, 355 